\title{
Primary epithelioid sarcoma of the vulva in pregnancy
}

\author{
Aydan Eroğlu $\cdot$ Aliye Sarı $\cdot$ Nazan Eroglu
}

Received: 4 February 2007 / Accepted: 5 July 2007 / Published online: 15 August 2007

(C) Springer-Verlag 2007

\begin{abstract}
Primary epithelioid sarcoma (ES) of the vulva is an extremely rare tumor, and to date, only 20 cases have been reported. We report the second case of vulvar ES presenting in pregnancy. A 25-year-old woman, at 6 weeks of gestation, was diagnosed as having vulvar sarcoma and was referred to our hospital. Immunohistochemical studies revealed that the tumor cells were positive for cytokeratin, epithelial membrane antigen (EMA), and wimentin. The tumor cells did not stain for S-100, SMA, HMB-45, desmin, LCA, CD-34, or estrogen and progesterone receptors. The tumor showed diffuse and strong staining for CD-117 (c-kit). After the curettage, she underwent local wide excision and left inguinal lymph node dissection and received external-beam radiation therapy. At 16 months of follow-up the patient was well without any disease recurrence. The appropriate treatment of vulvar ES is not clear, and the role of chemotherapy is disappointing; however, it might be worth investigating the role of Imatinib in c-kit overexpressing vulvar ES.
\end{abstract}

Keywords Epithelioid sarcoma $\cdot$ Vulva $\cdot$ Pregnancy

Treatment $\cdot$ C-kit

\footnotetext{
A. Eroğlu $(\bowtie)$

Department of General Surgery and Surgical Oncology,

Numune State Hospital Selçuklu,

Konya 42050, Turkey

e-mail: aydaneroglu@hotmail.com

A. Sar1

Department of Pathology, Numune State Hospital,

Konya, Turkey

N. Eroglu

Radiation Oncology Unit, Numune State Hospital,

Konya, Turkey
}

\section{Introduction}

Sarcomas are rare in the vulva, with leiomyosarcoma being the most common [1]. The occurrence of soft tissue sarcoma of the vulva in pregnancy is exceptional. A few cases have been reported that were associated with pregnancy [2-4]. Epithelioid sarcoma (ES) is extremely rare. Since Piver et al. first reported a case with ES in the vulva [5], 20 cases of vulvar ES have been published in the English literature [6-15]. One was diagnosed during pregnancy [13]. The clinical behavior of vulvar ES is not well understood because of the small number and short period of observation for some patients. The tumor shows different behavior, changing from an aggressive tumor to behaviors like those in low-grade tumors. Therefore, the optimal management of vulvar ES remains unknown.

In this report, a new case of ES of the vulva during pregnancy is presented, and the literature on the subject is reviewed with particular attention to its biological behavior and treatment.

\section{Case report}

A 25-year-old, gravida 2, at 6 weeks of gestation presented a slowly growing nodular mass in the left labium majus, and the lesion was locally excised at another hospital. The initial pathology report indicated vulvar sarcoma, and the representative paraffin block and slides were sent for revision to our pathology department. In the biopsy specimens, the tumor measured $4 \times 2 \mathrm{~cm}$ in size and $5 \mathrm{~mm}$ in depth. The lesion had an irregular border, with an ulceration of the overlying epidermis. Microscopically, the tumor was composed of nests of cells with multinodular appearance and tumor necrosis. The tumor cells were polygonal and 
epithelioid in shape and contained large pleomorphic nuclei with prominent nucleoli. The cytoplasm was large and eosinophilic. There were multinucleate giant cells and bizarre cells (Fig. 1). The tumor nodules were surrounded by chronic inflammatory cells. There was no sign of vascular invasion. The tumor was of intermediate grade. Mitotic count was three to four mitosis per ten high-power fields. One surgical margin had tumor cells and others were very close to the tumor.

Immunohistochemical stains were positive for cytokeratin, epithelial membrane antigen (EMA), and wimentin. The tumor cells did not stain for S-100, SMA, HMB-45, desmin, LCA, or CD-34. The tumor was estrogen- and progesterone-receptor negative. In addition, CD-117 (c-kit proto-oncogene product) was stained strong diffusely in the cytoplasm and/or cell membrane of the tumor cells (Fig. 2). On the basis of these pathologic findings, the tumor was diagnosed as vulvar ES.

After the pregnancy was terminated, the patient was referred to our surgical oncology unit for further definitive surgery. Physical examination of the external genital revealed only the presence of a small scar on the left labium major. An enlarged left superficial inguinal lymph node was found. Computed tomography (CT) of the chest, abdomen, and pelvis was negative for thorax, intraabdominal, or retroperitoneal disease. The patient underwent a clitoris-sparing wide local excision of the scar, with margins of at least $2 \mathrm{~cm}$ and left inguinal lymph node dissection. Pelvic lymphadenectomy was not performed because no pathological lymph node was detected on CT, and Cloquet's lymph node was found to be negative for malignant cells. Postoperative course was uneventful. Histological examination of the resected specimens showed no signs of residual tumor, and two of ten inguinal lymph nodes were metastatic.

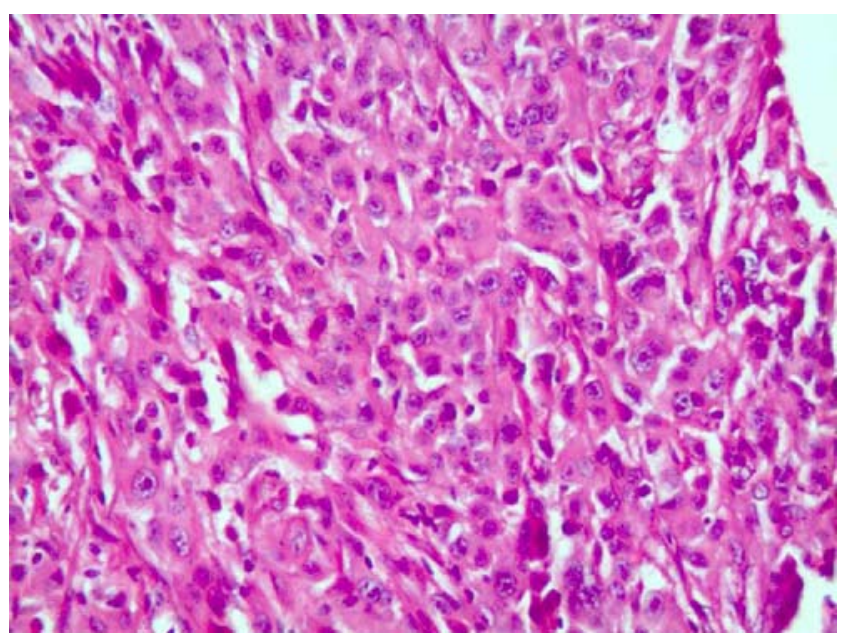

Fig. 1 Epithelioid sarcoma consisting of polygonal- and epithelioidshaped tumor cells with large eosinophilic cytoplasm, pleomorphic nuclei, and prominent nucleoli (hematoxylin and eosin stain)

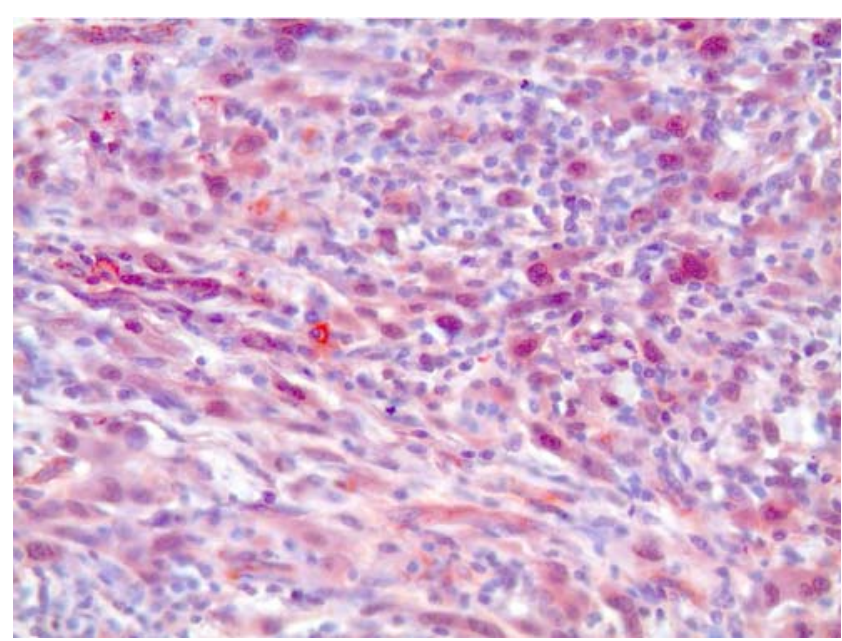

Fig. 2 Epithelioid sarcoma showing CD-117 reactivity in cytoplasmic and membranous staining pattern

The patient underwent adjuvant external beam radiotherapy, of $54 \mathrm{~Gy}$ in 30 fractions over 6 weeks, to the pelvis and inguinal areas, with central shielding of the vulva. The patient was well at the time of writing this report, and the follow-up examinations every 3 months revealed no evidence of disease recurrence.

\section{Discussion}

As first described in 1970, ES is usually located in distal and proximal extremities of young, particularly male, adults, and is differentiated from other neoplasms by findings of nodularity, frequent necrosis, acidophilic polygonal epithelioid cells in nests or sheets, and tendency for the tumor to form clefts [16]. The diagnosis of ES can be difficult due to histological confusion with other types of sarcomas. The differential diagnosis can be made with other tumors, such as epithelioid peripheral nerve sheath tumor (S100 positive), malignant melanoma (S100 and HMB-45 positive), epithelioid angiosarcoma (CD 31 positive), rhabdomyosarcoma (cytokeratin negative), and leiomyosarcoma (SMA positive) $[12,15,17]$. The occurrence of soft tissue sarcoma of the vulva in pregnancy is exceptional. Previously, Moore et al. reported the first case of ES in the vulva presenting during pregnancy [13]. To our knowledge, this is only the second case. The differential diagnosis of a vulvar mass in pregnancy includes leiomyoma, fibroma, Bartholin's gland cyst, and, less commonly, primary vulvar carcinoma and sarcoma. Therefore, the possible diagnosis of vulvar ES should be kept in mind upon presentation of a slowly growing nodular lesion.

The majority of ES occurs in distal extremities. In contrast to extremity, vulvar ES has been an aggressive tumor that metastasizes earlier $[6,7,9,12,13,15]$. 
Although local recurrence of ES is common, survival is related to distant metastasis. There is no adequate information from which to draw valid prognostic conclusions because of the small number and short period of observation for some patients. However, regional lymph node metastasis, presence of necrosis, vascular invasion, tumor size larger than $2 \mathrm{~cm}$, deep location, and high mitotic index in excess of two per ten high-power fields are poor prognostic factors [18]. In the view of the prognostic factors, our case had some poor prognostic factors, showing larger tumor size, high mitotic index, lymph node metastases, and presence of necrosis. The patient was well without disease recurrence at 16 months of follow-up.

Hormonal factors in the pathogenesis of ES are not established. As previously reported Moore et al., the lack of estrogen and progesterone receptors suggests the pregnancy does not necessarily accelerate the tumor growth, and steroid hormones may not affect tumor progression [13].

The optimal management of vulvar ES remains to be determined. Surgery varies from wide local excision to radical vulvectomy with inguinal lymph node dissection. Some authors suggest wide local excision $[5,8,10]$. Excision should include $2 \mathrm{~cm}$ from the tumor margin. On the other hand, some authors prefer radical vulvectomy to local excision [9, 12-15]. Because of the aggressive biology, poor outcome, and resistance to radiation and chemotherapy of ES, radical resection including inguinal lymph node dissection is suggested as appropriate treatment, except for early stage disease. Although ES has a high incidence of lymph node metastasis compared with most other sarcoma types [18], regional lymph node dissection for staging should be considered when they are suspicious or enlarged. Further research may be needed to establish the role of sentinel node biopsy in the management of patients with vulvar ES. Surgery, whenever offered, should be done with minimal morbidity.

Groin lymph node dissection should be performed in patients with clinically suspicious or positive lymph node, because there is no evidence of the beneficial effect of prophylactic lymph node dissection on local or distant recurrence. In our case, we performed the left inguinal lymph node dissection because of clinically enlarged lymph nodes at presentation.

The role of radiation and/or chemotherapy is not clear in patients with vulvar ES. Ross et al. suggested that radiation therapy had some benefits for the tumor greater than $5 \mathrm{~cm}$ [10]. Ulutin et al. stated that definitive surgery with adjuvant radiation therapy provided excellent tumor control in low-grade sarcomas [14]. However, radiation therapy can be recommended for cases with high-grade tumor or those with margin involvement or lymph node metastasis. Our patient received radiotherapy for nodal metastases to maximize local control.
In general, soft tissue sarcomas respond poorly to chemotherapy. In vulvar ES, chemotherapy has also been disappointing. The published patient reports have indicated that pulmonary metastasis has a very dismal prognosis, and chemotherapy adds little benefit to the treatment of the patients with metastatic disease $[9,13,15]$. Among human tumors, the c-kit proto-oncogene is frequent in gastrointestinal stromal tumors (GISTs); however; c-kit immunostaining has been found in some tumors other than GIST [19]. In our case, the tumor showed diffuse and generally strong staining for c-kit. Imatinib, a tyrosine kinase inhibitor to inhibit the activity of c-kit, has been used in the treatment GISTs [20]. The high frequency of diffuse positive staining for c-kit proto-oncogene product suggest that Imatinib may have a role in vulvar ES treatment as well as GIST.

In conclusion, vulvar ES is a very rare tumor, especially in pregnancy. The disease is best treated with early diagnosis and wide local excision with a margin of least $2 \mathrm{~cm}$. Although the role of chemotherapy is not clear in patients with advanced vulvar ES, it might be worth investigating if therapy against the c-kit, such as Imatinib, is effective for advanced vulvar ES that is c-kit positive.

\section{References}

1. Behranwala KA, Latifaj B, Blake P, Barton DPJ, Shepherd JH, Thomas JM (2004) Vulvar soft tisuue tumors. Int J Gynecol Cancer 14:94-99

2. Priore RM, Patton GD, Conti EA (1996) Pregnancy complicated by non-pigmented sarcoma of the vulva: Report of a case and review of the literature. Obstet Gynecol 27:420-427

3. Kuller JA, Zucker PK, Peng TC (1990) Vulvar leiomyosarcoma in pregnancy. Am J Obstet Gynecol 162:164-166

4. Di Gilio AR, Cormio G, Resta L, Carriero C, Loizzi V, Parisi AM, Selvaggi L (2004) Rapid growth of myxoid leiomyosarcoma of the vulva during pregnancy: A case report. Int J Gynecol Cancer 14:172-175

5. Piver MS, Tsukada Y, Barlow J (1972) Epithelioid sarcoma of the vulva. Obstet Gynecol 40:839-842

6. Hall DJ, Grimes MM, Goplerud DR (1980) Epithelioid sarcoma of the vulva. Gynecol Oncol 9:237-246

7. Ulbright TM, Brokaw SA, Stehman FB, Roth LM (1983) Epithelioid sarcoma of the vulva. Evidence suggesting a more aggressive behavior than extra-genital epithelioid sarcoma. Cancer 52:1462-1469

8. Tan GW, Lim-Tan SK, Salmon YM (1989) Epithelioid sarcoma of the vulva. Singapore Med J 30:308-310

9. Konefka T, Senkus E, Emerich J, Dudziak M (1994) Epithelioid sarcoma of the Bartholin's gland primarily diagnosed as vulvar carcinoma. Gynecol Oncol 54:393-395

10. Ross HM, Lewis JJ, Woodruff JM, Brennan MF (1997) Epithelioid sarcoma: Clinical behavior and prognostic factors of survival. Ann Surg Oncol 4:491-495

11. Guillou L, Wadden C, Coindre JM, Krausz T, Fletcher CD (1997) "Proximal-type" epithelioid sarcoma. A distinctive aggressive neoplasm showing rhabdoid features: Clinicopathologic, immunohistochemical, and ultrastructural study of a series. Am J Surg Pathol 21:130-146 
12. Kasamatsu T, Hasegawa T, Tsuda H, Okada S, Sawada M, Yamada T, Tsunematsu R, Ohmi K, Mizuguchi K, Kawana T (2001) Primary epithelioid sarcoma of the vulva. Int J Gynecol Cancer 11:316-320

13. Moore RG, Steinhoff MM, Granai CO, De Mars LR (2002) Vulvar epithelioid sarcoma in pregnancy. Gynecol Oncol 85:218222

14. Ulutin HC, Zellars RC, Frassica D (2003) Soft tissue sarcoma of the vulva: a clinical study. Int J Gynecol Cancer 13:528-531

15. Altundag K, Dikbas O, Oyan B, Usubutun A, Turker A (2004) Epithelioid sarcoma of vulva. A case report and review of the literature. Med Oncol 21:367-372

16. Enzinger FM (1970) Epithelioid sarcoma. A sarcoma simulating a granuloma or carcinoma. Cancer 26:1029-1041
17. Enzinger FM, Weiss SW (1994) Soft tissue tumors, 3rd edn. Mosby-Year Book, Missouri, pp 511-522

18. Chase DR, Enzinger FM (1985) Epithelioid sarcoma: Diagnosis, prognostic indicators, and treatment. Am J Surg Pathol 9:241-263

19. Heinrich MC, Blanke CD, Druker BJ, Corless CL (2002) Inhibition of KIT tyrosine kinase activity : a novel molecular approach to the treatment of KIT-positive malignancies. J Clin Oncol 20:1692-1703

20. Demetri GD, von Mehren M, Blanke CD, Van den Abbeele AD, Eisenberg B, Roberts PJ, Heinrich MC, Tuveson DA, Singer S, Janicek M, Fletcher JA, Silverman SG, Silberman SL, Capdeville R, Kiese B, Peng B, Dimitrijevic S, Druker BJ, Corless C, Fletcher CD, Joensuu H (2002) Efficacy and safety of imatinib mesylate in advanced gastrointestinal stromal tumors. N Engl $\mathrm{J}$ Med 347:472-480 